\title{
Time-Based Transition Expectancy in Task Switching: Do We Need to Know the Task to Switch to?
}

SPECIAL

COLLECTION:

THE STATE-OF-THEART OF RESEARCH

INTO HUMAN

MULTITASKING

RESEARCH ARTICLE

] $\mathrm{u}$ [ubiquity press

\section{ANDREA KIESEL (D) \\ ROLAND THOMASCHKE (D)}

*Author affiliations can be found in the back matter of this article

\begin{abstract}
Recent research has shown that humans are able to implicitly adapt to time-transition contingencies in a task-switching paradigm, indicated by better performance in trials where the task transition (switch vs. repetition) is validly predicted by the pre-target interval compared to trials with invalidly predicted transitions. As participants switched between only two different tasks, not only the transition, but also the specific task was predictable; at least indirectly when taking into account the temporally predicted transition in the current trial together with the task in the previous trial. In order to investigate if the time-based expectancy effect for transition in previous studies was due to a specific task preparation or due to an unspecific transition preparation, three different tasks were used in the present study. One of two possible pre-target intervals (500 and 1500 ms) predicted a task switch in the upcoming trial with $90 \%$ probability, whereas the other interval predicted a task repetition with $90 \%$ probability. Results revealed that participants were able to prepare both upcoming repetition as well as switch requirements based on predictive pre-target intervals. This means that humans seem to be able to prepare a task switch in a rather unspecific manner, most likely by inhibiting the task just performed in the previous trial. By suggesting a two-stage preparation model in which switches as well as repetitions benefit both from timebased transition expectancy, although apparently with different cognitive processes being involved, the present study provides important impulses for future research on the cognitive processes underlying human task-switching behavior.
\end{abstract}

CORRESPONDING AUTHOR:

\section{Stefanie Aufschnaiter}

Albert-Ludwigs-Universität Freiburg, Cognition, Action, and Sustainability Unit, Department of Psychology, Engelbergerstrasse 41, 79085 Freiburg, Germany stefanie.aufschnaiter@ psychologie.uni-freiburg.de

KEYWORDS:

Task switching; timing; timebased expectancy; preparation

TO CITE THIS ARTICLE: Aufschnaiter, S., Kiesel, A., \& Thomaschke, R. (2021). TimeBased Transition Expectancy in Task Switching: Do We Need to Know the Task to Switch to? Journal of Cognition, 4(1): 19, pp. 1-14. DOI: https://doi. org/10.5334/joc.145 
Every day we engage in multitasking, be it voluntarily or not (see also Himi, Bühner, Schwaighofer, Klapetek, \& Hilbert, 2019; Parry \& le Roux, 2018). In the laboratory, multitasking is typically investigated by applying either a dual task paradigm, where two tasks have to be processed concurrently (Pashler, 1994), or a task switching paradigm, where two or more tasks have to be executed in succession without temporal overlap (Kiesel et al., 2010; Vandierendonck, Liefooghe, \& Verbruggen, 2010; Ward, Hussey, Cunningham, Paul, McWilliams, \& Kramer, 2019, see Koch, Poljac, Müller \& Kiesel, 2018 for a recent combined discussion of both paradigms). The general finding in task switching studies is that switching to another task takes longer compared to the repetition of the task which has just been executed in the previous trial. This performance difference in reaction times (RTs) between task switches and task repetitions is usually referred to as switch costs (see Kiesel et al., 2010, for a review).

Recently, it has been shown that humans are able to make use of the predictive value of pretarget intervals in order to adjust their anticipatory cognitive control for preparing upcoming task execution requirements (Aufschnaiter, Kiesel, Dreisbach, Wenke, \& Thomaschke, 2018a; Aufschnaiter, Kiesel, \& Thomaschke, 2018b; Aufschnaiter, Kiesel, \& Thomaschke, 2020). Importantly, this enhanced preparation could not only be observed when the upcoming task identity was predicted based on time, but also when only the upcoming task transition (i.e. task switch vs. task repetition) was predicted based on the preceding time interval (Aufschnaiter et al., 2018a). However, as participants switched between only two different tasks in these studies, participants could actually still infer the specific task from the current interval and their knowledge about the previous task. Consequently, by using three tasks instead of two, the present study examines the question whether the time-based expectancy effect for transition is due to specific task preparation, or due to unspecific preparation of task transition.

\section{TIME-BASED TASK EXPECTANCY IN TASK SWITCHING}

There are several sources of predictability in the task switching paradigm which can be employed in order to adjust anticipatory cognitive control like, for instance, explicit task cues preceding the target stimuli (e.g. Dreisbach, Haider, \& Kluwe, 2002; Koch \& Allport, 2006) or explicit (Koch, 2005) and implicit (Gotler, Meiran, \& Tzelgov, 2003) task sequences. Thus, the predictive value of pre-target intervals is one among other potential sources of task predictability which can be used to enhance preparation for upcoming task requirements.

Task preparation based on duration information was first documented by Aufschnaiter et al. (2018a). They revealed that participants benefit not only from prolonging the pre-target interval (Meiran, 1996; Rogers \& Monsell, 1995), but that participants can make use of the predictive value of a pre-target interval in order to adjust anticipatory cognitive control regarding the upcoming task requirements. Most importantly, performance benefits in trials with validly predicted tasks compared to trials with invalidly predicted tasks were independent from the absolute length of the respective pre-target interval, and could be observed in a wide temporal range from 10 ms to even 3000 ms (Aufschnaiter et al., 2020).

The evidence for humans being able to implicitly adapt to time-task contingencies, and to show better performance in trials with validly predicted tasks compared to invalidly predicted tasks, is commonly explained by time-based expectancy as the underlying cognitive process (see Thomaschke \& Dreisbach, 2015, for a review). Time-based expectancy is usually investigated by applying the so-called time-event correlation paradigm, a specific variation of the foreperiod paradigm (Schröter, Birngruber, Bratzke, Miller, \& Ulrich, 2015), which was introduced by Wagener and Hoffmann (2010). In this paradigm, two pre-target intervals of different absolute length as well as two target stimuli appear equally often, but the combinations of interval and target differ regarding their overall frequency. The short interval is frequently combined with one target, whereas the long interval is frequently combined with the other target. It is assumed that participants learn the contingencies between intervals and targets implicitly by an associative learning mechanism (see Abrahamse, Braem, Notebaert, \& Verguts, 2016; Los, Kruijne, \& Meeter, 2014; Thomaschke $\&$ Dreisbach, 2015). The learned associations between intervals and targets are assumed to generate time-based expectancies which means that expectancy is directed towards the target associated with the short interval at the beginning of a trial. In case the short interval has passed without any target presentation, expectancy is directed towards the target associated with the long interval (see Aufschnaiter et al., 2018a; Thomaschke, Bogon, 
\& Dreisbach, 2018; Volberg \& Thomaschke, 2017). Consequently, time-based expectancy is typically indicated by better performance in trials with frequent combinations of interval target combinations compared to trials with infrequent interval - target combinations.

Please note, that in contrast to time-based expectancy, which means expecting a specific target at a certain point in time, time expectancy refers to as expecting when something will happen, independently from what exactly will happen at that point in time (Capizzi \& Correa, 2018; Grabenhorst, Michalareas, Maloney, \& Poeppel, 2019). Time expectancy is typically investigated by applying the above-mentioned foreperiod paradigm where the temporal distance between warning signal and target is manipulated (Los \& Agter, 2005; Steinborn \& Langner, 2012). However, time expectancy is conceptually different from time-based expectancy, and both forms of temporal expectancy usually do not interact (see Thomaschke $\&$ Dreisbach, 2015). For these reasons, time expectancy will not further be discussed in the present study.

\section{TIME-BASED TRANSITION EXPECTANCY IN TASK SWITCHING}

Interestingly, previous studies could show that not only preparation for specific task identities is enhanced by the adjustment of anticipatory cognitive control but also the preparation for task transitions (i.e. task switches and task repetitions; Bonnin, Gaonac'h, \& Bouquet, 2011; Dreisbach \& Haider, 2006, Farooqui \& Manly, 2015; Monsell \& Mizon, 2006).

For example, Dreisbach and Haider (2006) compared performance in experimental blocks with task switches in $75 \%$ of all trials (frequent-switch blocks) with performance in experimental blocks with task repetitions in $75 \%$ of all trials (frequent-repetition blocks). Interestingly, the authors found increased RTs for improbable task repetitions in frequent-switch blocks, especially when cues indicated the probability of a task switch before each trial. Moreover, a study by Farooqui and Manly (2015) revealed improved performance in trials with task switches, which had been announced by a subliminal cue at the beginning of a trial. The authors concluded that humans seem to be able to implicitly use subliminal information in order to adjust anticipatory cognitive control processes without being aware of it. Furthermore, it could be shown that participants implicitly make use of the predictive value of pre-target intervals in order to adjust their cognitive control involved in preparatory processes and to show better performance in trials with validly predicted task transitions compared to trials with invalidly predicted task transitions (Aufschnaiter et al., 2018a). In the study by Aufschnaiter et al. (2018a) one of two possible pre-target intervals (500 and $1500 \mathrm{~ms}$ ) predicted the upcoming task transition (task switch vs. task repetition) with $90 \%$ (Experiment 4) probability. Participants showed significantly faster RTs in trials with validly predicted task transitions compared to trials with invalidly predicted task transitions. Importantly, this time-based expectancy of task transition was not restricted to task switches but also task repetitions profited when validly predicted by time. Thus, results support the prevailing view in cognitive research on task switching that task repetitions require cognitive control processes as well (see Altmann, 2004; Dreisbach \& Haider, 2006; Koch, 2001).

However, there are two different possible explanations for the time-based expectancy effect for task transition in the experiments by Aufschnaiter and colleagues (2018a). On the one hand, the requirements of the specific task to be executed in the upcoming trial could have been either solely inferred from the pre-target interval being predictive about the upcoming task transition (direct unspecific preparation of task transition). On the other hand, it is also possible that the requirements of the specific task to be executed in the upcoming trial were inferred from both the predictive value of the pre-target interval and the knowledge about the previous task in trial n-1 (indirect specific task preparation).

Please note that the first account assuming unspecific preparation of a task transition suggests a role of backward inhibition of the task just executed (see Koch, Gade, Schuch, \& Philipp, 2010 for a review on the role of inhibition in task switching) ${ }^{1}$ : In case participants are indeed able

1 Backward inhibition in task switching has been majorly investigated by employing three different tasks. For example, Mayr and Keele (2000) found N-2 task repetition costs which were indicated by slower responses in the last trial of an ABA sequence compared to the last trial in a CBA sequence. The authors explained these $\mathrm{N}-2$ task repetition costs in the former case, which they called backward inhibition effect, with the inhibition of task $A$ once this task had been executed and is actively suppressed in order to perform task B. This inhibition of task A after having executed the $\mathrm{N}-2$ trial leads to worse performance once this task has to be performed again in an $A B A$ sequence (however, see Grange, Kowalczyk, \& O’Loughlin, 2017, for an alternative explanation). 
to prepare a task transition in a direct, rather unspecific manner, this would most likely mean that participants inhibit, at the point in time typical for switches, the task which had just been performed in the previous trial.

However, please note further that some studies suggested that participants cannot benefit from inhibiting the improbable task when being presented with probability cues about the task to be performed in the upcoming trial, if no specific information about the upcoming task requirements is provided (see Dreisbach et al., 2002; Mayr \& Keele, 2000; but see Chiu \& Egner, 2017 for an alternative explanation). Thus, these authors proposed that inhibition of the improbable task is only possible if specific information about the upcoming task requirements is available.

\section{RESEARCH QUESTION}

To examine the question whether the time-based preparation of a task transition is due to a specific task preparation or due to an unspecific preparation of a task transition, a combination of the standard task switching paradigm (cf. Kiesel et al., 2010) and the time-event correlation paradigm (Wagener \& Hoffmann, 2010) was used. The procedure involved two different intervals (500 ms and $1500 \mathrm{~ms}$ ) and three different tasks. One of the two intervals predicted a task switch in the upcoming trial with $90 \%$ probability, the other interval predicted a task repetition with $90 \%$ probability. Concerning time-based expectancy, three hypotheses can be formulated for this design:

(1) The design of the present study did not allow to infer specific information about the task requirements of switch trials: After having executed task $A$ in trial $n-1$, task $B$ as well as task $C$ were equiprobable when a switch was predicted by the pre-target interval in trial $n$ (see Figure 1). Nevertheless, in case participants are indeed able to prepare a task switch in a direct, but rather unspecific manner, and thus are capable of learning the association between interval and task transition, we would expect a significant time-based expectancy effect for task switches as well as for task repetitions. Such an unspecific preparation for a task switch in a paradigm with three tasks would most likely mean that participants inhibit, at the point in time typical for switches, the task which had just been performed in the previous trial (see Koch et al., 2010, for a review; Strobach, Wendt, \& Janczyk, 2018).

(2) If a task-specific preparation process is required for time-based transition expectancy, a time-based expectancy effect should not be observable for task switches in a paradigm with three different tasks. In case of a predicted task switch, the only information that can be derived is that the task of the previous trial will probably not occur in the next trial and each of the two tasks which have not been presented in the previous trial can occur with equal probability (see Figure 1). Thus, no time-based expectancy effect should emerge for task switches in case time-based transition expectancy implies a task-specific preparation process. However, in this case, a time-based transition expectancy effect should be observable for task repetitions. If the pre-target interval is predictive for a task repetition, the specific preparation of the upcoming task should be possible, as the task which has been performed in the previous trial can be specifically expected and is most likely to occur at that point in time (see Figure 1).

(3) However, it can also be the case that no effect at all can be found neither for task repetitions, nor for task switches, because a specific preparation of a task set would only be possible in half of the trials (when time predicts an upcoming task repetition) in the , 
present design. Thus, in case time-based transition expectancy implies a task-specific preparation, this time-based task-specific preparation would only be possible in $50 \%$ of all trials (as in trials with a predicted task switch, each of the two tasks which have not been presented in the previous trial can occur with equal probability and thus no task-specific preparation is possible; see Figure 1). Until now, no study has investigated if time-based expectancy is possible at all if only one of two intervals has a predictive value. Thus, it might be possible that participants do not learn the time-transition contingencies in this case or at least cannot make use of it.

As could be derived from the hypotheses, we foremost wanted to investigate, if participants are able to expect both, task switches as well as task repetitions, based on predictive intervals. Therefore, we included the within-subject factors "predictability of intervaltransition combination" and "task transition" in our analysis. If we would find a main effect for predictability of interval-transition combination and no interaction between predictability of interval-transition combination and transition, this would probably mean that hypothesis 1 would be supported by our data. However, in this case, we assumed inhibitory mechanisms to be involved in time-based expectancy for task switches, as no specific knowledge can be inferred about the task to be performed in the upcoming trial whenever a task switch is validly predicted based on time in a paradigm with three different tasks (see explanation of hypothesis 1 for detailed information). For this reason, we also included the between-subject factor "interval of expected repetition" in our analysis²: Whereas half of the participants expected a task repetition to occur frequently after $500 \mathrm{~ms}$ (group 1), the other half of the participants expected a task repetition to occur frequently after 1500 ms (group 2; see Figure 2). If humans are able to expect a task switch based on time in a rather generic manner without specific knowledge about the upcoming task, hypothesis 1 predicts that this would most likely mean that inhibitory processes might be involved. Thus, if inhibitory mechanisms are involved whenever a task switch is expected to occur after $500 \mathrm{~ms}$, participants in group 2 should inhibit the task, which had just been performed in the previous trial, during the first $500 \mathrm{~ms}$ of the current trial. However, if no stimulus is presented after 500 ms, which means that a task repetition is now to be expected based on the long interval, participants in this group would have to re-activate the task, which had just been inhibited (see Figure 2) and should thus show a smaller time-based expectancy effect for task repetitions compared to participants in group 1 , who expected a task repetition to occur after $500 \mathrm{~ms}$.

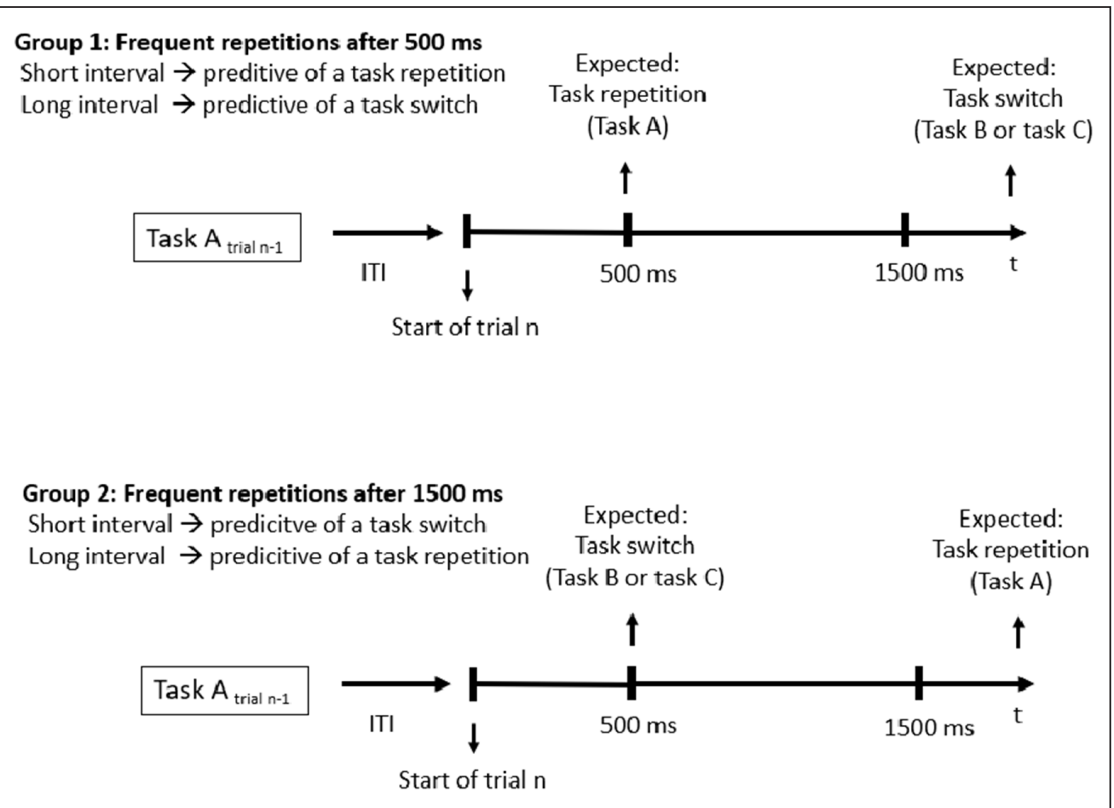

2 Considering the "interval of expected repetition" seems also interesting because of the following reason: In case of a short/long pre-target interval, we simultaneously introduce a variation of the response-to-stimulus interval (RSI) between trials, i.e. the respective RSI is short or long. Given that previous studies indicated that RSI manipulations impact on switch costs (e.g. Meiran, Chorev, \& Sapir, 2000), mainly by affecting performance in task repetitions (Horoufchin, Philipp, \& Koch, 2011), it seems reasonably to consider types of expectations for short/long intervals separately
Figure 2 Schematic illustration of the experimental betweensubject condition "interval of expected repetition": Whereas one half of participants (group 1) expected a task repetition to occur frequently after 500 ms in trial $\mathrm{n}$, the other half of participants (group 2) expected a task repetition to occur frequently after 1500 ms trial $n$. 
As our hypotheses also include potential null effects, we conducted a Bayesian ANOVA with default prior scales using JASP (JASP team, version 0.8.1.2) and looked at the Bayes tests for both the main effect of predictability of interval-transition combination (hypotheses 1 and 3 ) as well as for an interaction between transition and predictability of interval-transition combination (hypothesis 2).

\section{METHOD \\ PARTICIPANTS}

192 participants $\left(66\right.$ females $^{3}$; mean age $=29.99, S D=10.41$, range $=18-69$ years ${ }^{4} ; 162$ right handed ${ }^{5}$ ) took part in the experiment. Participants were recruited via the online participant recruitment tool Prolific (https://www.prolific.co/) and took part in exchange for monetary compensation. All participants spoke fluently English, had normal or corrected-to-normal vision, and were naive concerning the hypotheses. Participants provided written informed consent prior to the experiment. In order to make sure that participants fully concentrated on the experiment without any interruptions and followed the instruction to react as fast and as correctly as possible, we a priori excluded participants who showed an error rate above $25 \%$ and who took longer than one hour to complete a session. We suspected these participants of not having fully concentrated on the experiment without any interruptions or distractions and re-collected data for the respecting experimental conditions.

We used a stopping rule to determine the necessary sample size, and decided to stop data collection whenever the Bayes Factor (BF) would get larger than 3 either in favor of the null hypothesis $(\mathrm{HO})$ regarding the main effect of predictability (no difference of mean RTs between trials with predictable interval - transition combinations and trials with unpredictable intervaltransition combinations), or in favor of the alternative hypothesis $(\mathrm{H} 1)$. As the interaction between transition and predictability of interval-transition combination played a crucial role concerning our hypotheses, a further criterion for stopping data collection was that, in addition, the $\mathrm{BF}$ regarding the interaction of transition and predictability of interval-transition combination had to get larger than 3 either in favor of HO (no difference of mean RTs between switch and repetition trials regarding the predictability of interval-transition combinations), or in favor of $\mathrm{H} 1$. The Bayesian approach is a model selection procedure that indicates the likelihood ratio of two or more hypotheses based on the given data. Thus, Bayesian analysis provides the possibility of evaluating evidence in favor of the (null-) hypothesis. In this context, a BF in the range of 3 to 10 indicates moderate evidence in favor of the HO resp. $\mathrm{H} 1$ (Lee \& Wagenmakers, 2013). For reasons of counterbalancing, we first conducted the respective Bayes analyses after 64 participants, then after 128 participants and finally reached the stopping criteria after 192 participants.

\section{APPARATUS AND STIMULI}

Participants responded by key presses on the keyboard of their computer (keys $w$ and o; it was not allowed to use the mobile phone or a tablet in order to be eligible to take part in the study). Target stimuli were blue, green or red numbers between 1 and 9 , except 5 , presented against a black background (Arial typeface, font size $1.5 \mathrm{~cm}$ ). The fixation cross was the "+" symbol (Arial typeface, font size $1.5 \mathrm{~cm}$ ). All stimuli were presented centrally on the screen.

\section{PROCEDURE}

Each trial started with a blank screen for $300 \mathrm{~ms}$ (inter-trial interval, ITI), which was followed by the presentation of a white fixation cross for a variable interval of either $500 \mathrm{~ms}$ or $1500 \mathrm{~ms}$. After this interval, the target stimulus was presented. The order of stimuli was randomized, and each stimulus occurred with equal probability. Depending on the color of the target stimulus (blue, green or red), participants had to judge whether the displayed number was smaller or larger than 5 , whether it was odd or even, or whether the displayed number was distant to 5 (e.g. 1, 2, 8, 9) or near to 5 (e.g. 3, 4, 6, 7) on a mental number line. The latter task will be

\footnotetext{
One participant indicated "other".

One participant did not indicate his/her age.

Six participants indicated that they were ambidextrous.
} 
referred to as the inner-outer task from here on. The mapping of colors to tasks was held constant across participants. If the number was displayed in blue, participants had to perform the magnitude judgment task, and if the number was displayed in green, participants had to perform the parity judgment task. A red color indicated the inner-outer task. Responses were given with the same two buttons for all three tasks. The mapping of responses to keys was counterbalanced across participants. Participants were instructed to respond as fast and as correctly as possible. After the detection of an error, the word Error was displayed in white on a black screen for $1500 \mathrm{~ms}$. After correct responses, no explicit feedback was given. The duration of the interval predicted the upcoming task transition in the current trial. This means, a task switch occurred frequently after one interval, while a task repetition appeared frequently after the other interval. This means that when the interval was predictive of a task repetition after having executed task A, task A was predictable with $90 \%$ probability, whereas task B and task $C$ were each predictable with $5 \%$ probability. In contrast, when the interval was predictive of a task switch after having executed task $A$, task A was predictable with only $10 \%$ probability, whereas task B and Task $C$ were each predictable with $45 \%$ probability (see Figure 1). Both, the intervals, as well as the three tasks, appeared with the same overall frequencies. The mapping of task transition (switch vs. repetition) to interval was counterbalanced across participants. Participants were not informed that the intervals had different durations, or that these interval durations were correlated with task transitions.

The experiment consisted of two sessions of about 30 min each, which were tested on consecutive days. Both sessions of the experiment consisted of four blocks each: one learning block, and three test blocks. Each block comprised 120 trials. The only difference between learning blocks and test blocks was that after the detection of an error, the instruction was once again presented in white font color on a black screen for $8000 \mathrm{~ms}$ in the learning blocks, before the next trial started with the presentation of the ITI. Between blocks, participants could take a break, which they could terminate themselves by pressing the spacebar. After the second session of the experiment, participants had to complete a short survey in which they were asked if they had noticed any temporal regularities in the experiment.

\section{RESULTS}

Following earlier studies on time-based expectancy, we analyzed only the second session (e.g. Thomaschke \& Dreisbach, 2013). Data from the learning blocks, from the first three trials of each test block, as well as trials with number repetitions, and trials following an error trial were excluded from analyses. In addition, we excluded trials with RTs $<100 \mathrm{~ms}$ from analyses and we removed trials, in which RTs deviated more than 3 standard deviations from the corresponding cell mean, computed separately for each participant, block and experimental condition (Bush, Hess, \& Wolford, 1993). Furthermore, trials with errors were removed from the RT analyses.

Based on the sample of 192 participants, we conducted an outlier analyses and excluded three participants from the following analyses due to high error rates in comparison to the other participants (mean percentage of errors for the three excluded participants: $9.2 \%, 9.8 \%$ and $15.8 \%$; mean percentage of errors for all participants: $2.40 \%$ ). This resulted in a final sample of 189 participants.

First, we conducted a three-factor ANOVA with the within-subjects factors transition (switch vs. repetition), predictability of interval - transition combination (predictable vs. unpredictable) and the between-subjects factor "interval of expected repetition", meaning that we took into account if a task repetition was expected after the short or after the long pre-target interval, dependent of the respective experimental condition (for a detailed description of this analysis in theoretical terms, see Introduction). Additionally, we performed Bayesian ANOVA with default prior scales using JASP. Please notice that during the following analyses, we will explicitly mention whenever BF and $p$-value do not support each other, and that we will further indicate explicitly, whenever there was only anecdotal Bayesian evidence in favor of $\mathrm{HO}$ or $\mathrm{H1}$ (in accordance to Lee \& Wagenmakers, 2013).

RTs and error rates are displayed in Figure 3. Results revealed a significant main effect for transition, meaning that responses were faster in trials with task repetitions $(M=1073 \mathrm{~ms}$, 
$S D=477)$ compared to task switches $\left(M=1311^{6}, S D=417\right), F(1,187)=83.476, p<.001, \eta_{p}{ }^{2}$ $=.309$. Moreover, the main effect for predictability of interval-transition combination gained significance, $F(1,187)=12.891, p<.001, \eta_{p}{ }^{2}=.064$, meaning that responses in trials with predictable interval-transition combinations were faster $(M=1158 \mathrm{~ms}, S D=359)$ than in trials with unpredictable interval-transition combinations ( $M=1226 \mathrm{~ms}, S D=492)$, BF in favor of H1 $=4.159$. Whereas the interaction between transition and "interval of expected repetition" did not turn significant, $F(1,187)<1, p=.559, \eta_{p}{ }^{2}=.002$, BF in favor of $\mathrm{HO}=7.220$, the interaction between predictability of interval-transition combination and "interval of expected repetition" turned significant, $F(1,187)=4.555, p=.034, \eta_{p}{ }^{2}=.024$, BF in favor of $\mathrm{H} 1=0.450$ (please note that $p$-value and BF did not support each other and that we will not further interpret this interaction in the general discussion for this reason). The interaction between transition and predictability of interval-transition was not significant, $F(1,187)=1.460, p=.229, \eta_{p}^{2}=$ $.008, \mathrm{BF}$ in favor of $\mathrm{HO}=3.453$, but, however, the interaction between "interval of expected repetition", transition and predictability of interval-transition combination turned significant, $F(1,187)=3.950, p=.048, \eta_{p}{ }^{2}=.021, \mathrm{BF}$ in favor of $\mathrm{H} 1=1.982$ (anecdotal evidence, see Lee $\&$ Wagenmakers, 2013). The main effect of the between-subjects factor "interval of expected repetition" did not turn significant, $F(1,187)<1, p=.582, \eta_{p}{ }^{2}=.002$, BF in favor of HO = 4.623.

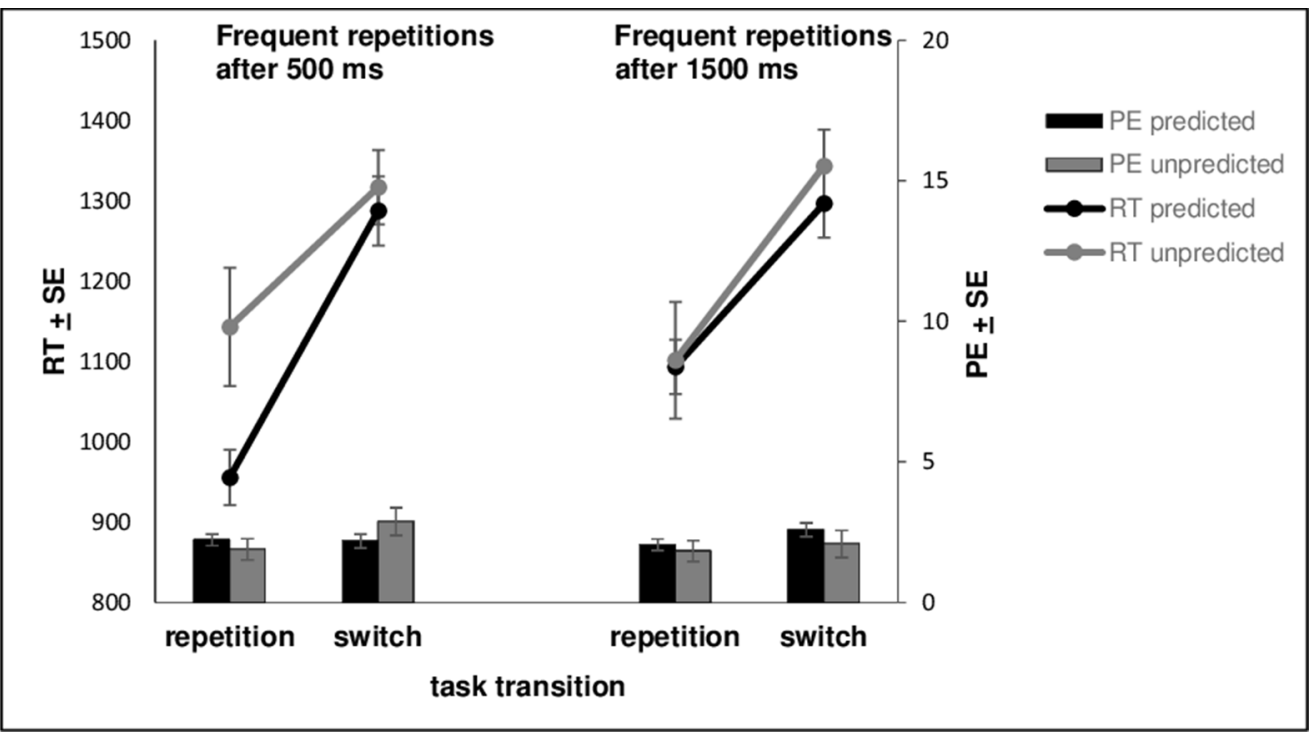

Given the significant three-way interaction for RTs, we conducted two ANOVAs with the withinsubject factor predictability of interval-transition combination (predictable vs. unpredictable) and the between-subject factor interval of expected repetition (expected repetitions after 500 ms, group 1, vs. expected repetitions after 1500 ms, group 2; see also Figure 2) separately for task switches and for task repetitions.

For task switches, we observed a significant main effect for predictability of interval-transition combination, $F(1,187)=6.077, p=.015, \eta_{p}^{2}=.031$, BF in favor of $\mathrm{H} 1=2.108$ (anecdotal evidence, see Lee \& Wagenmakers, 2013), meaning that responses in trials with predictable interval-transition combinations were significantly faster $(M=1292 \mathrm{~ms}, S D=414)$ compared to responses in trials with unpredictable interval-transition combinations $(M=1330 \mathrm{~ms}, S D=$ 446). The main effect of "interval of expected repetition", $F(1,187)<1, p=.771, \eta_{p}{ }^{2}=.000$, $\mathrm{BF}$ in favor of $\mathrm{HO}=2.540$ (anecdotal evidence, see Lee \& Wagenmakers, 2013), as well as the interaction between predictability of interval-transition combination and "interval of expected repetition" did not turn significant, $F(1,187)<1, p=.578, \eta_{p}{ }^{2}=.002$, BF in favor of $\mathrm{HO}=5.525$. In group 1 (expected repetitions after $500 \mathrm{~ms}$ ), responses were faster in trials with predictable interval-transition combinations $(M=1287 \mathrm{~ms}, S D=591)$ compared to trials with unpredictable combinations of interval-transition combinations $(M=1317 \mathrm{~ms}, S D=635)$ and this was also the case for participants in group 2 (expected repetitions after $1500 \mathrm{~ms} ; M=1296 \mathrm{~ms}$, SD $=581$ 464, in trials with predictable interval-transition combinations vs. $M=1343 \mathrm{~ms}, S D=625464$, in trials with unpredictable interval-transition combinations).
Figure 3 Mean reaction times (RTs in ms; lines) and percentages of errors (PES in \%; bars), depending on predictability of interval transition combination, are displayed separately for task transition and the betweensubject condition "interval of expected task repetition" (500 ms vs. 1500 ms). Error bars represent 1 standard error of the mean. 
For task repetitions, we also observed a significant main effect for predictability of intervaltransition combination, $F(1,187)=5.642, p=.019, \eta_{p}^{2}=.029$, BF in favor of $\mathrm{H} 1=1.421$ (anecdotal evidence, see Lee \& Wagenmakers, 2013), meaning that responses in trials with predictable interval-transition combinations were significantly faster $(M=1024 \mathrm{~ms}, S D=331)$ compared to responses in trials with unpredictable interval-transition combinations $(M=1122 \mathrm{~ms}, S D=$ 711). The main effect of "interval of expected repetition", $F(1,187)<1, p=.489, \eta_{p}{ }^{2}=.003$, BF in favor of $\mathrm{HO}=4.418$, did not turn significant. However, the interaction between predictability of interval-transition combination and "interval of expected repetition" turned significant, $F$ $(1,187)=4.720, p=.031, \eta_{p}^{2}=.025, \mathrm{BF}$ in favor of $\mathrm{H} 1=1.348$ (anecdotal evidence, see Lee \& Wagenmakers, 2013). In group 1 (expected repetitions after $500 \mathrm{~ms}$ ), responses were faster in trials with predictable interval-transition combinations $(M=955 \mathrm{~ms}, S D=471)$ compared to trials with unpredictable interval-transition combinations $(M=1143 \mathrm{~ms}, S D=1014), t(92)$ $=-.2 .285, p=.025$. However, for participants in group 2 (expected repetitions after $1500 \mathrm{~ms}$ ), this difference in RTs was much smaller $(M=1093 \mathrm{~ms}, S D=464$, in trials with predictable interval-transition combinations vs. $M=1102 \mathrm{~ms}, S D=998$, in trials with unpredictable intervaltransition combinations), $t(95)=-.508, p=.613$.

For error rates, results revealed no significant main effects or interactions for the ANOVA with the within-subjects factors transition and predictability of interval-transition combination and the between-subjects factor "interval of expected repetition" (max. $F=3.560$, $\min . p=0.61$ ).

Only three of the 192 participants noticed temporal regularities during the course of the experiment, as they stated in the survey after the second session that sometimes, numbers occurred more quickly than others, and hence facilitated expectations for the same color to appear again.

\section{GENERAL DISCUSSION}

The present study investigated whether the benefit from time-based transition expectancy in task switching (cf. Aufschnaiter et al., 2018a) is due to an unspecific preparation of a task transition or rather due to a specific task preparation by inferring task identity based on the previous task identity and the prediction of the task transition in trials with validly predicted switches, when only two different tasks are used (cf. Aufschnaiter et al., 2018a). For this purpose, the present study employed three different tasks instead of two (cf. Aufschnaiter et al., 2018a). One of two possible intervals (500 vs. $1500 \mathrm{~ms}$ ) predicted a task switch in the upcoming trial with $90 \%$ probability, the other interval predicted a task repetition with $90 \%$ probability.

Our results revealed a significant time-based expectancy effect, which was further supported by Bayesian analysis, and importantly, this effect did not interact with task transition (switch vs. repetition). Thus, the assumption of unspecific preparation of a task transition seems to be supported by the present data: As the design of the present study did not allow to infer specific information about the task requirements of switch trials, our results indicate at first glance that participants seem to be able to learn the association between the pre-target interval and task transition and thus seem to prepare a task switch in a direct, but rather unspecific manner.

However, please note that we found a significant three-way interaction between transition, predictability of interval-transition combination and interval of expected repetition (i.e. group), which was supported by at least anecdotal Bayesian evidence. The between-subject factor group meant that we took into account, if participants belonged to the group, which expected task repetitions to occur frequently after $500 \mathrm{~ms}$ (group 1), or if they belonged to the group, which expected task repetitions to occur frequently after the long interval (group 2). Importantly, when solely investigating task switches based on the significant three-way interaction, we found a significant predictability effect, which was, though, supported by at least anecdotal evidence given our Bayesian analysis. However, the predictability effect for task switches did not interact with group, which was further supported by Bayesian analyses. This means that based on these additional analyses we can infer that task switches really seem to benefit from time-based predictability and that participants seem to be able to prepare a task switch in a rather unspecific manner without knowledge about the specific requirements of the upcoming task. 
Assuming that participants benefitted from inferring specific knowledge about the task to be performed in the upcoming trial when a task repetition was validly predicted based on duration information (see also Koch, 2008), the question remains, though, which cognitive preparatory mechanism supports switch preparation based on time. As it was already stated above, an unspecific preparation for a task switch in a paradigm with three tasks would most likely mean that participants inhibit, at the point in time typical for switches, the task that had just been performed in the previous trial (see Koch et al., 2010, for a review). And importantly, this is exactly what our data suggest: When only investigating task repetitions based on the significant three-way interaction, we find a significant interaction between predictability of intervaltransition combination and group, for which the corresponding Bayesian analysis revealed at least anecdotal evidence. In group 1, where participants expected a task repetition to occur frequently after the short interval of $500 \mathrm{~ms}$, we find a time-based expectancy effect for task repetitions, whereas in group 2 , where participants expected task repetitions to occur frequently after the long interval of $1500 \mathrm{~ms}$, no time-based expectancy effect for task transitions could be observed. While task switches seem to benefit from time-based expectancy in both groups of participants (see above), participants obviously could only prepare for a task repetition based on a predictive interval, when belonging to group 1, where they expected task repetitions to occur frequently after the short interval of $500 \mathrm{~ms}$, explaining the significant three-way interaction. However, as already stated above, this finding, which may come unexpected at first sight, provides first evidence for the account of inhibitory processes underlying time-based expectancy for task transition.

Let us assume that the task, which had just been performed, is inhibited when a switch is predicted based on time after the short interval of $500 \mathrm{~ms}$. This means that participants, who expect a switch to occur frequently after 500 ms should inhibit the task just performed in the previous trial during the first $500 \mathrm{~ms}$ of the current trial. When no stimulus appears after $500 \mathrm{~ms}$, they have to re-direct their expectancy in the direction of a task repetition, which is likely to appear after the long interval of $1500 \mathrm{~ms}$. However, this would mean that they would have to "re-activate" the inhibited task, which should imply a time-consuming cognitive effort. And indeed, as it was explained above, for participants who expected task switches to occur after 500 ms, we observed, even numerically, no expectancy effect when a task repetition occurred after 1500 ms (even if the appearance of a task repetition after 1500 ms implied a predictable interval-transition combination and should thus lead to performance benefits). This observation speaks in favor of inhibitory processes being involved in time-based expectancy for task switches, which cannot be easily overcome if expectancy has to be re-directed from an expected task switch towards an expected task repetition.

Though, previous studies suggested that the requirement for inhibition is that the upcoming task can be specifically prepared, be it a task switch or a task repetition (see Dreisbach et al., 2002). However, in the present study, a task could only be specifically prepared if the interval predicted a task repetition: In this case, the task which had been just performed in the previous trial was likely to occur in the following trial with $90 \%$ probability. As our results also revealed a time-based expectancy effect for task switches, where no explicit information about the specific task requirements of the upcoming switch trial could be derived, the present study questions previous studies which stated that inhibition processes require the possibility of specific task preparation, and rather suggests an unspecific switch preparation whenever a task switch is validly predicted based on time.

Based on previous studies, suggesting cognitive preparatory processes, which take place both in repetition and switch trials (see Dreisbach et al., 2002; Koch, 2005), we suggest a two-stage preparation model in which switches as well as repetitions benefit both from time-based transition expectancy. In this context, two-stage models of task set reconfiguration in switch trials have been proposed by numerous studies (see Kiesel et al, 2010; Koch et al., 2018): The first stage (the so-called "advance reconfiguration", Rogers \& Monsell, 1995; "goal shifting", Rubinstein, Meyer, \& Evans, 2001) contributes to preparation for the upcoming cognitive requirements before target presentation. The second stage ("rule activation", Koch et al., 2018) can only be passed if the specific target stimulus is provided. Although two-stage models are usually discussed in the context of upcoming switch requirements, we propose that two different preparatory stages might also play a role regarding time-based expectancy of task repetitions: Whenever a task repetition is validly predicted by time, the first stage might involve a specific task preparation, by inferring knowledge about the identity of the task just performed 
in the previous trial (as the explicit task cue, the color, is not presented until target onset). The possibility for specific task preparation during the first stage of the preparation process might account for the at least numerically larger expectancy effect for task repetitions after $500 \mathrm{~ms}$ in group 1, where task repetitions were expected to occur frequently after $500 \mathrm{~ms}$, compared to task switches after 500 ms in group 2, where task switches were expected to occur frequently after 500 ms (see Figures 2 and 3). Moreover, our results suggest that participants are able to prepare also task switches based on a predictive pre-target interval and thus, also the first preparation stage before target onset in switch trials seems to benefit from time-based expectancy. Based on our conclusions, on which we elaborated above, we suggest that in case a task switch is validly predicted based on time, the first preparation stage for expected task switches might involve the inhibition of the task just performed and might allow to implement an unspecific switch readiness.

Furthermore, regarding future studies, our results offer implications for studies in the context of hierarchical task switching (see Schneider \& Logan, 2006). Future studies should, for example, investigate, if participants can benefit from time-based transition expectancy, when an interval is not only predictive of the requirement to switch on a single trial basis (as it was the case in the present study) but when it is predictive of the frequency of switches in a whole task sequence. In this context, a recent study by Chiu and Egner (2017) seems to provide important implications regarding future research on time-based transition expectancy in task switching: The authors found that humans are able to associate certain stimuli with increased switch requirements and show reduced switch costs when being presented with these stimuli. Most importantly, by employing three different tasks (as it was the case in the present study) the authors assume that a general switch readiness as a cognitive bottom-up process is possible, which has nothing to do with the preparedness to switch to an alternative task, but rather with a state of increased cognitive flexibility. As in the study of Chiu and Egner (2017) switch readiness was induced on the level of single trials, indicated by item-specific switch probability (ISSP) effects, it would indeed be interesting to induce switch readiness for longer task sequences based on predictive intervals in order to investigate if switch readiness can be built up in a rather flexible manner between whole task sequences. Interestingly, a recent study by Liu and Yeung (2019) investigated the influence of explicit instructions about switch requirements in an upcoming task sequence and revealed evidence that it is possible to induce different meta-control states which can be adapted given different probabilities of frequent-switch sequences compared to infrequent-switch sequences. However, please notice that in the study of Liu and Yeung (2019), participants were explicitly instructed about switch requirements in an upcoming task sequence. To our knowledge, no study has ever investigated before if it is possible to prime a global switch readiness regarding upcoming task execution requirements in whole sequences in an implicit manner (as it would be the case when predicting the switch likelihood in upcoming task sequences based on predictive intervals). Interestingly, the fact that previous studies could show that participants are able to use contextual information to prepare for an increase of upcoming task switches and that they maintain this information across many trials indicates a sort of higher-level cognitive control (see Braver, Reynolds, \& Donaldson, 2003; Dreisbach \& Haider, 2006), which may speak in favor of the possibility of inducing time-based transition expectancy on a higherorder sequence level.

Taken together, the present results revealed evidence that humans are able to prepare a task switch in a rather unspecific manner whenever a task switch is validly predicted by the pretarget interval, most likely by inhibiting the task just performed in the previous trial. Moreover, a time-based expectancy effect was also observed for task repetitions in the present study, probably by inferring specific knowledge about the identity of the task just performed in the previous trial when a task repetition was validly predicted based on time. We suggest a twostage preparation model in which switches as well as repetitions benefit both from time-based transition expectancy, although apparently with different cognitive processes being involved. Furthermore, by demonstrating that humans seem to be able to prepare for task switches in a direct, unspecific way without being presented with specific knowledge about the upcoming task requirements, the present results stimulate the highly topical research field of task switching by challenging future research on the cognitive processes underlying human task switching behavior and additionally provoke relevant impulses regarding future investigations of time-based expectancy. 


\section{DATA ACCESSIBILITY STATEMENT}

Raw data of the reported experiment are available via the Open Science Framework, DOI: 10.17605/OSF.IO/Q7RT2.

\section{ETHICS AND CONSENT}

All procedures performed in studies involving human participants were in accordance with the ethical standards of the institutional and/or national research committee and with the 1964 Helsinki declaration and its later amendments or comparable ethical standards.

Informed consent was obtained from all individual participants included in the study.

\section{ACKNOWLEDGEMENTS}

This research was supported by grants within the Priority Program, SPP 1772 from the German Research Foundation (Deutsche Forschungsgemeinschaft, DFG), grant no TH 1554/3-1 and TH 1554/3-2. We thank Younes Strittmatter for his support in programming the experiment.

\section{COMPETING INTERESTS}

The authors have no competing interests to declare.

\section{AUTHOR AFFILIATIONS}

Stefanie Aufschnaiter (D) orcid.org/ 0000-0003-4896-7360

Cognition, Action, and Sustainability Unit, Department of Psychology, University of Freiburg, Freiburg im Breisgau, DE

Andrea Kiesel (1D) orcid.org/0000-0001-5564-010X

Cognition, Action, and Sustainability Unit, Department of Psychology, University of Freiburg, Freiburg im Breisgau, DE

Roland Thomaschke (D) orcid.org/0000-0003-1251-4183

Cognition, Action, and Sustainability Unit, Department of Psychology, University of Freiburg, Freiburg im Breisgau, DE

\section{REFERENCES}

Abrahamse, E., Braem, S., Notebaert, W., \& Verguts, T. (2016). Grounding cognitive control in associative learning. Psychological Bulletin, 142(7), 693-728. DOI: https://doi.org/10.1037/bul0000047

Altmann, E. M. (2004). Advance preparation in task switching: What work is being done? Psychological Science, 15(9), 616-622. DOI: https://doi.org/10.1111/j.0956-7976.2004.00729.x

Aufschnaiter, S., Kiesel, A., Dreisbach, G., Wenke, D., \& Thomaschke, R. (2018a). Time-based expectancy in temporally structured task switching. Journal of Experimental Psychology: Human Perception and Performance, 44(6), 856-870. DOI: https://doi.org/10.1037/xhp0000494

Aufschnaiter, S., Kiesel, A., \& Thomaschke, R. (2018b). Transfer of time-based task expectancy across different timing environments. Psychological Research, 82(1), 230-243. DOI: https://doi.org/10.1007/ s00426-017-0895-1

Aufschnaiter, S., Kiesel, A., \& Thomaschke, R. (2020). Humans derive task expectancies from sub-second and supra-second interval durations. Psychological Research, 84(5), 1333-1345. DOI: https://doi. org/10.1007/s00426-019-01155-9

Bonnin, C. A., Gaonac'h, D., \& Bouquet, C. A. (2011). Adjustments of task-set control processes: Effect of task switch frequency on task-mixing and task-switching costs. Journal of Cognitive Psychology, 23(8), 985-997. DOI: https://doi.org/10.1080/20445911.2011.594435

Braver, T. S., Reynolds, J. R., \& Donaldson, D. I. (2003). Neural mechanisms of transient and sustained cognitive control during task switching. Neuron, 39(4), 713-726. DOI: https://doi.org/10.1016/S08966273(03)00466-5

Bush, L. K., Hess, U., \& Wolford, G. (1993). Transformations for within-subject designs: A Monte Carlo investigation. Psychological Bulletin, 113(3), 566-579. DOI: https://doi.org/10.1037/00332909.113.3.566

Capizzi, M., \& Correa, Á. (2018). Measuring Temporal Preparation. In A. Vatakis, F. Balcı, M. Di Luca, \& Á. Correa (Eds.), Timing and Time Perception: Procedures, Measures, \& Applications (pp. 216-232). Brill. DOI: https://doi.org/10.1163/9789004280205_011 
Chiu, Y. C., \& Egner, T. (2017). Cueing cognitive flexibility: Item-specific learning of switch readiness. Journal of Experimental Psychology: Human Perception and Performance, 43(12), 1950-1960. DOI: https://doi.org/10.1037/xhp0000420

Dreisbach, G., \& Haider, H. (2006). Preparatory adjustment of cognitive control in the task switching paradigm. Psychonomic Bulletin \& Review, 13(2), 334-338. DOI: https://doi.org/10.3758/ BF03193853

Dreisbach, G., Haider, H., \& Kluwe, R. H. (2002). Preparatory processes in the task-switching paradigm: Evidence from the use of probability cues. Journal of Experimental Psychology: Learning, Memory, and Cognition, 28(3), 468-483. DOI: https://doi.org/10.1037/0278-7393.28.3.468

Farooqui, A. A., \& Manly, T. (2015). Anticipatory control through associative learning of subliminal relations: invisible may be better than visible. Psychological Science, 26(3), 325-334. DOI: https://doi. org/10.1177/0956797614564191

Gotler, A., Meiran, N., \& Tzelgov, J. (2003). Nonintentional task set activation: Evidence from implicit task sequence learning. Psychonomic Bulletin \& Review, 10(4), 890-896. DOI: https://doi.org/10.3758/ BF03196549

Grabenhorst, M., Michalareas, G., Maloney, L. T., \& Poeppel, D. (2019). The anticipation of events in time. Nature Communications, 10, 5802. DOI: https://doi.org/10.1038/s41467-019-13849-0

Grange, J. A., Kowalczyk, A. W., \& O'Loughlin, R. (2017). The effect of episodic retrieval on inhibition in task switching. Journal of Experimental psychology: Human Perception and Performance, 43(8), $1568-$ 1583. DOI: https://doi.org/10.1037/xhp0000411

Himi, S. A., Bühner, M., Schwaighofer, M., Klapetek, A., \& Hilbert, S. (2019). Multitasking behavior and its related constructs: Executive functions, working memory capacity, relational integration, and divided attention. Cognition, 189, 275-298. DOI: https://doi.org/10.1016/j.cognition.2019.04.010

Horoufchin, H., Philipp, A. M., \& Koch, I. (2011). The dissipating task-repetition benefit in cued task switching: Task-set decay or temporal distinctiveness? Journal of Experimental Psychology: Human Perception and Performance, 37(2), 455-472. DOI: https://doi.org/10.1037/a0020557

Kiesel, A., Steinhauser, M., Wendt, M., Falkenstein, M., Jost, K., Philipp, A. M., \& Koch, I. (2010). Control and interference in task switching - A review. Psychological Bulletin, 136(5), 849-874. DOI: https:// doi.org/10.1037/a0019842

Koch, I. (2001). Automatic and intentional activation of task sets. Journal of Experimental Psychology: Learning, Memory, and Cognition, 27(6), 1474-1486. DOI: https://doi.org/10.1037/0278-7393.27.6.1474

Koch, I. (2005). Sequential task predictability in task switching. Psychonomic Bulletin \& Review, 12(1), 107-112. DOI: https://doi.org/10.3758/BF03196354

Koch, I. (2008). Instruction effects in task switching. Psychonomic Bulletin \& Review, 15(2), 448-452. DOI: https://doi.org/10.3758/PBR.15.2.448

Koch, I., \& Allport, A. (2006). Cue-based preparation and stimulus-based priming of tasks in task switching. Memory \& Cognition, 34(2), 433-444. DOI: https://doi.org/10.3758/BF03193420

Koch, I., Gade, M., Schuch, S., \& Philipp, A. M. (2010). The role of inhibition in task switching: A review. Psychonomic Bulletin \& Review, 17(1), 1-14. DOI: https://doi.org/10.3758/PBR.17.1.1

Koch, I., Poljac, E., Müller, H., \& Kiesel, A. (2018). Cognitive structure, flexibility, and plasticity in human multitasking - An integrative review of dual-task and task-switching research. Psychological bulletin, 144(6), 557-583. DOI: https://doi.org/10.1037/bul0000144

Lee, M. D., \& Wagenmakers, E. J. (2013). Bayesian data analysis for cognitive science: A practical course. New York: Cambridge University Press.

Liu, C., \& Yeung, N. (2019). Dissociating expectancy-based and experience-based control in task switching. Journal of Experimental Psychology: Human Perception and Performance. 46(2), 131-154. DOI: https://doi.org/10.1037/xhp0000704

Los, S. A., \& Agter, F. (2005). Reweighting sequential effects across different distributions of foreperiods: Segregating elementary contributions to nonspecific preparation. Perception \& Psychophysics, 67(7), 1161-1170. DOI: https://doi.org/10.3758/BF03193549

Los, S. A., Kruijne, W., \& Meeter, M. (2014). Outlines of a multiple trace theory of temporal preparation. Frontiers in Psychology, 5, 1058. DOI: https://doi.org/10.3389/fpsyg.2014.01058

Mayr, U., \& Keele, S. W. (2000). Changing internal constraints on action: The role of backward inhibition. Journal of Experimental Psychology: General, 129(1), 4-26. DOI: https://doi.org/10.1037//00963445.129.1.4

Meiran, N. (1996). Reconfiguration of processing mode prior to task performance. Journal of Experimental Psychology: Learning, Memory, \& Cognition, 22(6), 1423-1442. DOI: https://doi.org/10.1037/02787393.22.6.1423

Meiran, N., Chorev, Z., \& Sapir, A. (2000). Component processes in task switching. Cognitive Psychology, 41(3), 211-253. DOI: https://doi.org/10.1006/cogp.2000.0736

Monsell, S., \& Mizon, G. A. (2006). Can the task-cuing paradigm measure an endogenous task-set reconfiguration process? Journal of Experimental Psychology: Human Perception and Performance, 32(3), 493-516. DOI: https://doi.org/10.1037/0096-1523.32.3.493 
Parry, D. A., \& le Roux, D. B. (2018). Media multitasking and cognitive control: A systematic review of interventions. Computers in Human Behavior. DOI: https://doi.org/10.1016/j.chb.2018.11.031

Pashler, H. (1994). Dual-task interference in simple tasks: data and theory. Psychological Bulletin, 116(2), 220-244. DOI: https://doi.org/10.1037/0033-2909.116.2.220

Rogers, R. D., \& Monsell, S. (1995). Costs of a predictable switch between simple cognitive tasks. Journal of Experimental Psychology: General, 124(2), 207-231. DOI: https://doi.org/10.1037/00963445.124.2.207

Rubinstein, J. S., Meyer, D. E., \& Evans, J. E. (2001). Executive control of cognitive processes in task switching. Journal of Experimental Psychology: Human Perception and Performance, 27, 763-797. DOI: https://doi.org/10.1037/0096-1523.27.4.763

Schneider, D. W., \& Logan, G. D. (2006). Hierarchical control of cognitive processes: switching tasks in sequences. Journal of Experimental Psychology: General, 135(4), 623-640. DOI: https://doi. org/10.1037/0096-3445.135.4.623

Schröter, H., Birngruber, T., Bratzke, D., Miller, J., \& Ulrich, R. (2015). Task predictability influences the variable foreperiod effect: Evidence of task-specific temporal preparation. Psychological Research, 79(2), 230-237. DOI: https://doi.org/10.1007/s00426-014-0550-z

Steinborn, M. B., \& Langner, R. (2012). Arousal modulates temporal preparation under increased time uncertainty: Evidence from higher-order sequential foreperiod effects. Acta Psychologica, 139(1), 65-76. DOI: https://doi.org/10.1016/j.actpsy.2011.10.010

Strobach, T., Wendt, M., \& Janczyk, M. (2018). Multitasking: executive functioning in dual-task and task switching situations. Frontiers in Psychology, 9, 108. DOI: https://doi.org/10.3389/fpsyg.2018.00108

Thomaschke, R., Bogon, J., \& Dreisbach, G. (2018). Timing affect: Dimension-specific time-based expectancy for affect. Emotion, 18(5), 646-669. DOI: https://doi.org/10.1037/emo0000380

Thomaschke, R., \& Dreisbach, G. (2013). Temporal predictability facilitates action, not perception. Psychological Science, 24(7), 1335-1340. DOI: https://doi.org/10.1177/0956797612469411

Thomaschke, R., \& Dreisbach, G. (2015). The time-event correlation effect is due to temporal expectancy, not to partial transition costs. Journal of Experimental Psychology: Human Perception and Performance, 41(1), 196-218. DOI: https://doi.org/10.1037/a0038328

Vandierendonck, A., Liefooghe, B., \& Verbruggen, F. (2010). Task switching: Interplay of reconfiguration and interference control. Psychological Bulletin, 136(4), 601-626. DOI: https://doi.org/10.1037/ a0019791

Volberg, G., \& Thomaschke, R. (2017). Time-based expectations entail preparatory motor activity. Cortex, 92, 261-270. DOI: https://doi.org/10.1016/j.cortex.2017.04.019

Wagener, A., \& Hoffmann, J. (2010). Temporal cueing of target-identity and target-location. Experimental Psychology, 57(6), 436-445. DOI: https://doi.org/10.1027/1618-3169/a000054

Ward, N., Hussey, E. K., Cunningham, E. C., Paul, E. J., McWilliams, T., \& Kramer, A. F. (2019). Building the multitasking brain: An integrated perspective on functional brain activation during task-switching and dual-tasking. Neuropsychologia, 132. DOI: https://doi.org/10.1016/j. neuropsychologia.2019.107149
TO CITE THIS ARTICLE:

Aufschnaiter, S., Kiesel, A., \& Thomaschke, R. (2021). TimeBased Transition Expectancy in Task Switching: Do We Need to Know the Task to Switch to? Journal of Cognition, 4(1): 19, pp. 1-14. DOI: https://doi. org/10.5334/joc.145

Submitted: 26 January 2020 Accepted: 30 December 2020 Published: 10 March 2021

COPYRIGHT:

(c) 2021 The Author(s). This is an open-access article distributed under the terms of the Creative Commons Attribution 4.0 International License (CC-BY 4.0), which permits unrestricted use, distribution, and reproduction in any medium, provided the original author and source are credited. See http:// creativecommons.org/ licenses/by/4.0/.

Journal of Cognition is a peerreviewed open access journal published by Ubiquity Press. 